\title{
A future large-aperture UVOIR space observatory: reference designs
}

\author{
Harley Thronson*a, Norman Rioux ${ }^{\mathrm{a}}$, Lee Feinberg ${ }^{\mathrm{a}}$, H. Phillip Stahl ${ }^{\mathrm{b}}$, Dave Redding ${ }^{\mathrm{c}}$, Andrew \\ Jones $^{\mathrm{a}}$, James Sturm ${ }^{\mathrm{a}}$, Christine Collins ${ }^{\mathrm{a}}$, Alice Liu ${ }^{\mathrm{a}}$ \\ ${ }^{a}$ NASA Goddard Space Flight Center, Greenbelt, MD; ${ }^{b}$ NASA Marshall Space Flight Center, \\ Huntsville, AL, USA; 'Jet Propulsion Laboratory, CA, USA
}

\begin{abstract}
Our joint NASA GSFC/JPL/MSFC/STScI study team has used community-provided science goals to derive mission needs, requirements, and candidate mission architectures for a future large-aperture, non-cryogenic UVOIR space observatory. We describe the feasibility assessment of system thermal and dynamic stability for supporting coronagraphy. The observatory is in a Sun-Earth L2 orbit providing a stable thermal environment and excellent field of regard. Reference designs include a 36-segment $9.2 \mathrm{~m}$ aperture telescope that stows within a five meter diameter launch vehicle fairing. Performance needs developed under the study are traceable to a variety of reference designs including options for a monolithic primary mirror.
\end{abstract}

Keywords: ATLAST, LUVOIR, HDST, design concept, exo-planets, space telescope

\section{INTRODUCTION}

In the two decades since the discovery was reported of the first planet around a Sun-like star, 51 Pegasi, the study of exoplanets has progressed rapidly. The 2010 NRC Astronomy and Astrophysics Decadal Survey elevated the search for exoEarths to a major science goal and technology investment for a mission to search for exo-Earths as its highest-priority "Medium Activity" for the decade. NASA's Kepler mission, launched in 2009, has demonstrated that the Milky Way is teeming with planets, very likely - albeit not yet certainly - with millions of planets similar to the Earth.

As of this writing, there are now almost 2000 confirmed exo-planets and roughly another 3000 candidate exo-planets waiting additional data. Among these are some 300 objects lying within the so-called "habitable zone" around the central star. That is, perhaps around $10 \%$ or more of the stars in the sky have a planet roughly similar to the Earth at a location neither too hot nor too cold for life to arise.

In parallel with the growing recognition that Earth-like worlds may be abundant has been increasingly detailed study of a future space observatory capable of both distinguishing an exo-planet from its central star and suppressing the intense starlight that would otherwise overwhelm the faint planetary light. We will describe here the conceptual design work related to such a facility.

In addition to the capability to analyze the spectra of exo-planets and search for indicators of a biologically active world, the rest of the astronomical community has identified major scientific goals in the UV/optical/IR wavelength regimes. NASA's 30-year vision for astrophysics, Enduring Quests, Daring Visions, last year highlighted a Large UV/Optical/IR (LUVOIR) observatory as a priority mission for the 2020s. More recently, the Associated Universities for Research in Astronomy (AURA), which operates the Space Telescope Science Institute, recommended to the scientific community a similar concept, the High-Definition Space Telescope (HDST) in its new report, From Cosmic Birth to Living Earths ${ }^{1}$.

From the 2010 NRC Decadal Survey's priority recommendation to begin investments that would enable the search for exo-Earths, to NASA's future LUVOIR concept and AURA's HDST, successive studies have together identified a mission consistent with the design that we describe here. The conceptual design work described here applies to the ATLAST, LUVOIR and HDST missions. For simplicity of reference, the single acronym “ATLAST” appears below, but when it is used here it is intended as equally applicable to the science objectives referenced by LUVOIR and HDST.

*harley.a.thronson@nasa.gov; phone 301 286-5155 
The ATLAST concept was submitted to the 2010 Decadal Survey by a consortium of GSFC, MSFC, JPL and STScI. The proposal included a survey of three alternative design concepts, including observatories based around an $8 \mathrm{~m}$ monolith, $9.2 \mathrm{~m}$ segmented deployed primary mirror, and a $16 \mathrm{~m}$ primary mirror. The ATLAST proposal was well received by the 2010 Decadal Survey. As indicated above, the Decadal Survey elevated technology investment for a mission to search for exo-Earths as its highest-priority "Medium Activity" for the decade. In this paper, brief descriptions and references are provided for observatory design concepts based around the $8 \mathrm{~m}$ monolith primary mirror and a $12.7 \mathrm{~m}$ deployed primary mirror. This paper addresses aspects of mechanical and thermal stability for the conceptual design of an observatory with a scalable segmented primary mirror.

\section{SCIENCE OBJECTIVES}

The formative science requirements for the ATLAST mission are summarized in Tables 1 and 2 shown below. These parameters drive the development of engineering conceptual designs. In order to enable breakthroughs in astrophysics and astronomy, an aperture size greater than 8 meters in diameter is needed. Wave front error stability requirements for exoplanet science conducted with an internal coronagraph also stand out as driving the conceptual design in particular. More will be said later about the science objectives as they are examined for implementation with conceptual engineering designs. These science objectives are responsive to a broad program of interest to the science community at large. The objectives and parameters below are consistent with the community-provided science goals that appear in the Association of Universities for Research in Astronomy (AURA) report.

Table 1. ATLAST Telescope Parameter Table

\begin{tabular}{|c|c|c|c|c|}
\hline \multicolumn{2}{|c|}{ Parameter } & Requirement & Stretch Goal & Traceability \\
\hline \multicolumn{2}{|c|}{ Primary Mirror Aperture } & $\geq 8$ meters & 12 meters & $\begin{array}{l}\text { Resolution, Sensitivity, } \\
\text { Exoplanet Yield }\end{array}$ \\
\hline \multicolumn{2}{|c|}{ Telescope Temperature } & $273 K-293 K$ & - & $\begin{array}{l}\text { Thermal Stability, } \\
\text { Integration \& Test, } \\
\text { Contamination, } \\
\text { IR Sensitivity }\end{array}$ \\
\hline \multirow{4}{*}{$\begin{array}{l}\text { Wavelength } \\
\text { Coverage }\end{array}$} & UV & $100 \mathrm{~nm}-300 \mathrm{~nm}$ & $90 \mathrm{~nm}-300 \mathrm{~nm}$ & \\
\hline & Visible & $300 \mathrm{~nm}-950 \mathrm{~nm}$ & - & \\
\hline & NIR & $950 \mathrm{~nm}-1.8 \mu \mathrm{m}$ & $950 \mathrm{~nm}-2.5 \mu \mathrm{m}$ & \\
\hline & MIR & - & $\begin{array}{c}\text { Sensitivity to } 5.0 \mu \mathrm{m} \\
\text { under evaluation }\end{array}$ & Transit Spectroscopy \\
\hline \multirow{2}{*}{$\begin{array}{l}\text { Image } \\
\text { Quality }\end{array}$} & UV & $<0.20$ arcsec at $150 \mathrm{~nm}$ & - & \\
\hline & Vis/NIR/MIR & Diffraction-limited at $500 \mathrm{~nm}$ & - & \\
\hline \multicolumn{2}{|c|}{ Stray Light } & $\begin{array}{l}\text { Zodi-limited between } \\
400 \mathrm{~nm}-1.8 \mu \mathrm{m}\end{array}$ & $\begin{array}{l}\text { Zodi-limited between } \\
200 \mathrm{~nm}-2.5 \mu \mathrm{m}\end{array}$ & $\begin{array}{l}\text { Exoplanet Imaging \& } \\
\text { Spectroscopy SNR }\end{array}$ \\
\hline \multicolumn{2}{|c|}{ Wavefront Error Stability } & $\begin{array}{l}\sim 10 \text { pm RMS uncorrected } \\
\text { system WFE per control step }\end{array}$ & - & $\begin{array}{c}\text { Starlight Suppression } \\
\text { via Internal } \\
\text { Coronagraph }\end{array}$ \\
\hline \multirow{2}{*}{ Pointing } & Spacecraft & $\leq 1$ milli-arcsec & - & \\
\hline & Coronagraph & $<0.4$ milli-arcsec & - & \\
\hline
\end{tabular}


Table 2. ATLAST Instrument Parameter Table

\begin{tabular}{|c|c|c|c|}
\hline Science Instrument & Parameter & Requirement & Stretch Goal \\
\hline \multirow{3}{*}{$\begin{array}{l}\text { UV Multi-Object } \\
\text { Spectrograph }\end{array}$} & Wavelength Range & $100 \mathrm{~nm}-300 \mathrm{~nm}$ & $90 \mathrm{~nm}-300 \mathrm{~nm}$ \\
\hline & Field-of-View & $1-2 \operatorname{arcmin}$ & \\
\hline & Spectral Resolution & $\begin{array}{l}\mathrm{R}=20,000-300,000 \\
\text { (selectable) }\end{array}$ & \\
\hline \multirow{3}{*}{ Visible-NIR Imager } & Wavelength Range & $300 \mathrm{~nm}-1.8 \mu \mathrm{m}$ & $300 \mathrm{~nm}-2.5 \mu \mathrm{m}$ \\
\hline & Field-of-View & $4-8$ arcmin & \\
\hline & Image Resolution & $\begin{array}{l}\text { Nyquist sampled at } \\
500 \mathrm{~nm}\end{array}$ & \\
\hline \multirow{3}{*}{$\begin{array}{l}\text { Visible-NIR } \\
\text { Spectrograph }\end{array}$} & Wavelength Range & $300 \mathrm{~nm}-1.8 \mu \mathrm{m}$ & $300 \mathrm{~nm}-2.5 \mu \mathrm{m}$ \\
\hline & Field-of-View & $3-4 \operatorname{arcmin}$ & \\
\hline & Spectral Resolution & $\mathrm{R}=100-10,000$ (selectable) & \\
\hline \multirow{4}{*}{$\begin{array}{l}\text { MIR Imager / } \\
\text { Spectrograph }\end{array}$} & Wavelength Range & & $2.5 \mu \mathrm{m}-8 \mu \mathrm{m}$ \\
\hline & Field-of-View & & $3-4 \operatorname{arcmin}$ \\
\hline & Image Resolution & & $\begin{array}{l}\text { Nyquist sampled at } 3 \\
\mu \mathrm{m}\end{array}$ \\
\hline & Spectral Resolution & & $\mathrm{R}=5-500$ (selectable) \\
\hline \multirow{5}{*}{$\begin{array}{c}\text { Starlight Suppression } \\
\text { System }\end{array}$} & Wavelength Range & $400 \mathrm{~nm}-1.8 \mu \mathrm{m}$ & $200 \mathrm{~nm}-2.5 \mu \mathrm{m}$ \\
\hline & Raw Contrast & $1 \times 10^{-10}$ & \\
\hline & Contrast Stability & $1 \times 10^{-11}$ over integration & \\
\hline & Inner-working angle & 34 milli-arcsec @ 1 mm & \\
\hline & Outer-working angle & $>0.5$ arcsec @ $1 \mu \mathrm{m}$ & \\
\hline \multirow{2}{*}{$\begin{array}{l}\text { Multi-Band Exoplanet } \\
\text { Imager }\end{array}$} & Field-of-View & $\sim 0.5$ arcsec & \\
\hline & Resolution & $\begin{array}{l}\text { Nyquist sampled at } \\
500 \mathrm{~nm}\end{array}$ & \\
\hline \multirow{2}{*}{$\begin{array}{c}\text { Exoplanet } \\
\text { Spectrograph }\end{array}$} & Field-of-View & $\sim 0.5$ arcsec & \\
\hline & Resolution & $\mathrm{R}=70-500$ (selectable) & \\
\hline
\end{tabular}

\section{LAUNCH VEHICLE CONSIDERATIONS}

The ATLAST mission boldly sets out to enable scientific breakthroughs with the largest telescope aperture ever deployed in space. ATLAST is moving forward with a sense of urgency to provide these breakthroughs in the era of the 2020s and early 3030s. In the more distant future, it is reasonable to expect that larger and larger telescopes will be assembled in space. For ATLAST, the most economical and immediate path forward is to put a large aperture in space with a single launch.

The ATLAST observatory will be in a Sun-Earth L2 orbit providing a stable thermal environment and excellent field of regard. Constraints that limit the size of the telescope aperture include the amount of mass to orbit and the size of the fairing that the launcher can provide. Of paramount concern alongside enabling a large aperture is controlling mission cost and risk. The ATLAST mission seeks to be ready for a new start in the mid-2020s. The launch vehicle industry in that era will not be exactly the same as it is now, and changes can stem from unpredictable events. In order to control launch vehicle risk and its associated costs, ATLAST will have flexibility and robustness in being able to use a variety of different launch vehicles and fairing sizes.

It is clear that a mission with the ambitious goals of ATLAST will need a heavy lift launch vehicle. Figure 1 below depicts a variety of launch vehicles and lift capabilities. Figure 2 below depicts the fairing diameters of these launch vehicles. By designing the mission to be compatible with the variety of launch vehicles discussed in the paragraph below, ATLAST will have the strength to manage risks and costs with a flexible suite of alternatives. 


\section{Approximate Launch Mass to C3 $-0.5\left(\mathrm{~km}^{2} / \mathrm{sec}^{2}\right)$}

Mass to Orbit in Thousands of $\mathrm{kg}$
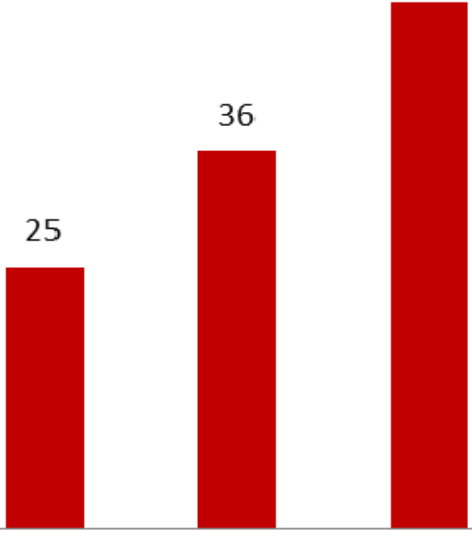

Falcon 9 v1.1 Atlas V (551) Ariane

Delta IV H

Falcon $\mathrm{H}$

SLS Block 1 SLS Block 1B SLS Block 2B

Figure 1. Approximate Launch Vehicle Mass to L2 Orbit

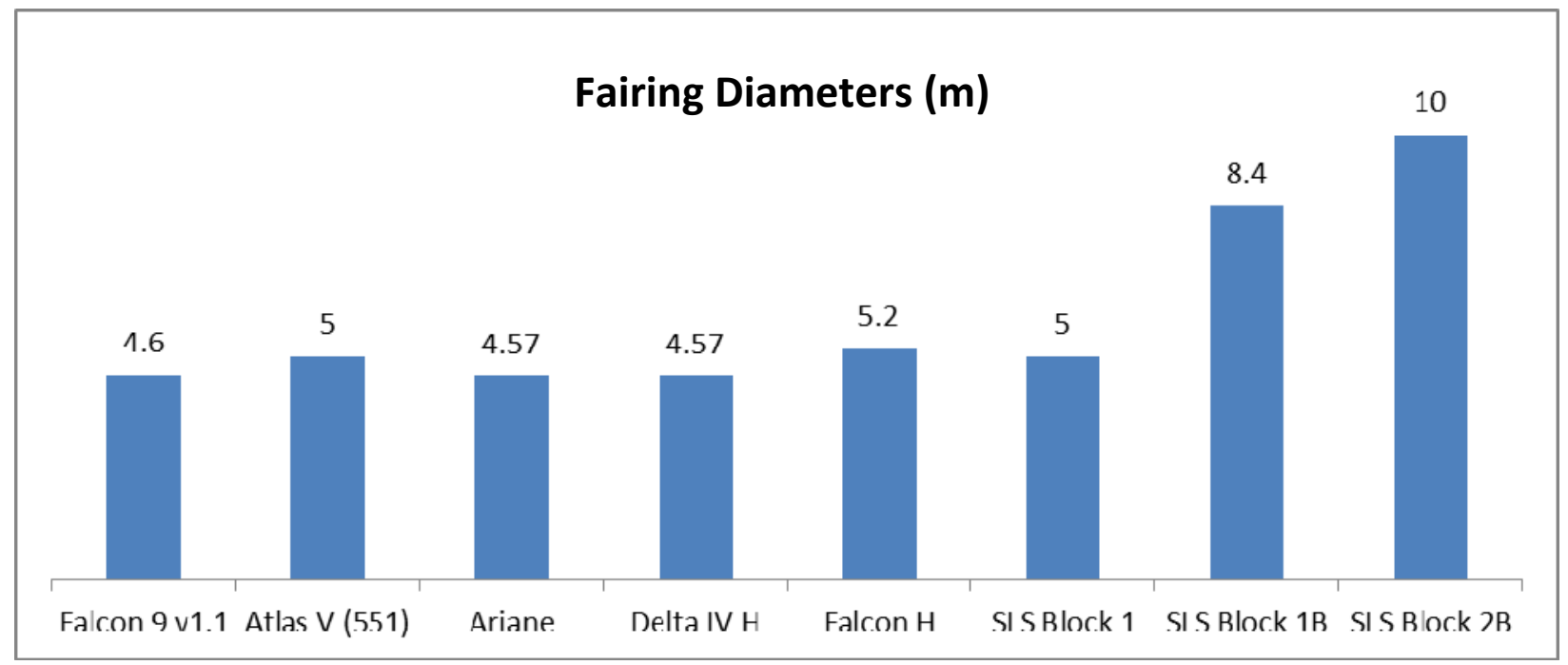

Figure 2. Launch Vehicle Fairing Diameters

The Delta IV Heavy is an existing heavy lift launch vehicle with a proven track record. Five meter launch vehicle fairings are a standard in the industry, and the Delta IV Heavy has a five meter fairing. ATLAST will substantiate its immediate readiness for selection to the 2020 Decadal Survey by having a design option that is ready to fly on an existing, proven launch vehicle. Compatibility with the proven Delta IV Heavy achieves this purpose. United Launch Alliance (ULA) has stated that they will continue to manufacture the Delta IV Heavy for as long as the Air Force wants it. ULA has also stated that they intend to build a heavy lift launch vehicle as the successor to the Delta IV Heavy to compete with the Falcon Heavy. The Falcon Heavy is in development by Space $\mathrm{X}$ and offers a lift capability in excess of the Delta IV Heavy and will have a five meter fairing. The Falcon Heavy is expected to be relatively economical, with launch costs currently projected to be on the order of \$100 M. The Space Launch System (SLS) is currently in development and will have unsurpassed mass to orbit capabilities, even in excess of the Falcon Heavy. Options are under study for $5 \mathrm{~m}, 8.4 \mathrm{~m}$, and $10 \mathrm{~m}$ fairings. ATLAST and SLS have already instituted engineer-to-engineer working group 
meetings to develop conceptual designs for interfaces between the observatory and the SLS launch vehicle. Concepts are being studied that will allow ATLAST to be compatible with any of the SLS launch vehicle fairings. The SLS will provide unsurpassed mass margin and the potential for unrivaled fairing volume margin for ATLAST. This can provide substantial risk mitigation, flexibility and robustness to the development of the ATLAST observatory.

\section{STARLIGHT SUPPRESSION TECHNIQUES}

ATLAST has co-emphasis on exoplanet studies and ultraviolet, optical and near IR survey astronomy. The identification of biomarkers on exoplanets endows the ATLAST mission with compelling and potentially revolutionary achievements in understanding the fundamental question of "Are we alone?" The exoplanet aspects of the mission contains some unique driving requirements, so early emphasis has been on accommodating these aspects of the mission. A key architectural feature of ATLAST is the starlight suppression system. At the conceptual level, this could be achieved with a free-flying star shade, or with a coronagraph that is internal to the observatory. Each of these approaches has their own strengths and challenges.

A free-flying star shade tends to impose less stringent requirements on the stability of the telescope, but it could take on the order of weeks to re-position from one target to another due to its significant distance from the telescope observatory. This in turn would drive down observing efficiency and exoplanet yield. On the other hand, a star shade can be a useful tool for in-depth study of an exoplanet that has been previously identified for study by other means.

An internal coronagraph repoints easily with the telescope that it is installed in. For this reason, it can be useful for exoplanet surveys and achieving a larger exoplanet yield. Coronagraphs tend to place more stringent requirements on telescope stability in ways that free flying star shades do not. Both star shades and coronagraphs are currently the subject of technology development programs and their design and performance characteristics are becoming more mature. ATLAST trade studies will continue to track their progress over the years ahead. Exoplanet yield tends to be a dominant science consideration for gathering statistics to characterize potentially life-bearing exo-planets. For that reason there is early emphasis on ATLAST mission concepts that use a coronagraph. Each of the engineering design concepts discussed later are initially focused on including an internal coronagraph for exoplanet science.

\section{TECHNOLOGY DEVELOPMENT}

ATLAST has developed a robust technology development plan that identifies technologies necessary for enabling the science mission. The technology development plan is described in more detail in the SPIE paper titled "Technology Development for the Advanced Technology Large Aperture Space Telescope (ATLAST) as a Candidate Large UVOptical-Infrared (LUVOIR) Surveyor" by M. R. Bolcar et al. ${ }^{2}$. Part of the role of the ATLAST engineering conceptual designs is to provide detailed means of connecting the science objectives to technologies necessary to enable meeting those objectives.

\section{ENGINEERING DESIGN REFERENCE MISSIONS}

ATLAST has been conceived with a vision for creating breakthrough, revolutionary science. Paired with that vision is emphasis on cost control and cost effectiveness as paramount priorities. A key to formulating the most cost effective ATLAST mission possible is the derivation and validation of detailed, verifiable requirements. Detailed requirements decomposed and flowed down to the lowest level of assembly drive the mission implementation, and the implementation drives costs. Implementing the system and verifying the implementation against the requirements also drives the schedule and the critical path. The critical path will have a dominant effect on the mission cost.

The purpose of the ATLAST Engineering Design Reference Missions (EDRMs) are to provide a basis for deriving a validated set of mission requirements from the science objectives and top level science requirements. The EDRMs allow the engineering design trade space to be explored in depth and the determination in detail where the stressing requirements are and where there are opportunities for margin against requirements. The EDRMs provide access to a rich trade space where implementations and requirements can be analyzed and evaluated against each other to formulate the most effective, well balanced, and lowest risk designs. In a perfect world, multiple EDRMs would be developed simultaneously in parallel and in depth to make the most progress the quickest in mapping out the science objectives against the requirements, implementation space, and costs. Since mission formulation must be done within the limitations of available resources, there are practical limits to how many in-depth EDRMs are currently being developed in parallel. 
Two of the key words in "Engineering Design Reference Mission" are "reference” and "design”. The EDRMs are not the final mission design. The EDRMs are conceptual designs used for deriving and validating requirements. Settling on "the design" in pre-phase A would be a recipe for misunderstood requirements and cost growth as the true requirements get discovered on a reactive basis on the project critical path. ATLAST will be designed to requirements that have been validated on a system wide, end to end basis. Validated mission requirements will open up the field for implementers to propose the most cost effective methods possible for delivering results against the mission requirements. With validated requirements in hand, opportunities will be created for implementers in industry, academia and government organizations to create and bring forward their best ideas to support the most cost effective mission implementation. Validated mission requirements enable robust trades studies to be performed that will help determine the most cost effective mission implementation.

\section{ATLAST CONCEPTUAL DESIGN STUDIES}

Multiple EDRMs have been generated for ATLAST. These include observatories based around an $8 \mathrm{~m}$ monolith primary mirror, a monolith surrounded by deployable mirror petals, and a segmented deployable mirror. Each of these represent different approaches to achieving the necessary aperture and system stability for the ATLAST mission. Each of them needs more conceptual design work to quantify their feasibility, performance, and cost effectiveness.

The $8 \mathrm{~m}$ monolith EDRM was generated in the 2009 timeframe and submitted to the 2010 Decadal Survey along with two design concepts using segmented deployable mirrors. These concepts were evaluated by the 2010 Decadal Survey and won the recommendation that technology development for a future observatory capable of studying Earth-like planets be matured for consideration by the 2020 Decadal Survey. The $8 \mathrm{~m}$ monolith concept is described in reference 3, published by Stahl et al. The $8 \mathrm{~m}$ monolith offers the advantage of not having segmentation gaps in the primary mirror. Deployable primary mirrors enable aperture sizes greater than the diameter of the launch vehicle fairing, however they necessitate gaps between segments in the deployed primary mirror. A monolith without gaps provides advantages with regard to some current coronagraph designs that are in development. An $8 \mathrm{~m}$ monolithic mirror would uniquely require the SLS Block II launch vehicle with a ten meter fairing. This vehicle is slated for development, but there would be no alternative means of launching the mission if this particular variant of the SLS launch vehicle does not materialize, creating a unique mission risk. Since new discoveries in astrophysics tend to be photon-limited, it is reasonable to expect that future space telescope mission will need larger apertures than ATLAST, and monolithic primary mirrors do not present a path into the future. The $8 \mathrm{~m}$ monolith EDRM provides a layout of the conceptual design space that would use the largest diameter monolithic primary mirror for a space application.

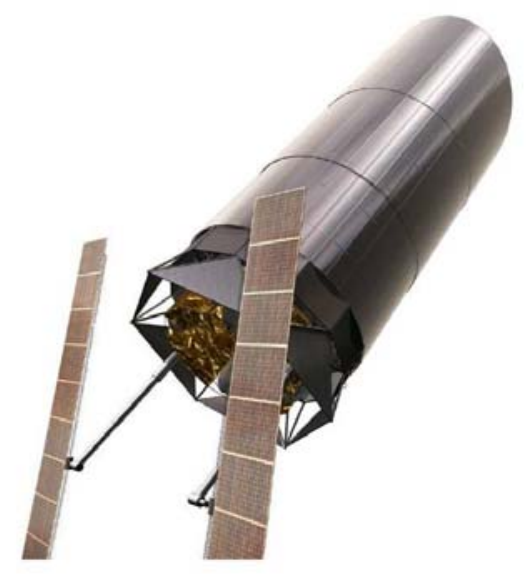

Figure 3. ATLAST design concept utilizing an $8 \mathrm{~m}$ monolithic primary mirror.

The $12.7 \mathrm{~m}$ EDRM considers a monolithic primary mirror surrounded by deployable mirror petals that extend the aperture to $12.7 \mathrm{~m}$. This is based on an analysis determining the largest diameter telescope with a center core and a single ring of segments that packages inside the mass and volume of an SLS launch vehicle with a $10 \mathrm{~m}$ diameter fairing. Figure 4 is a conceptual illustration of the telescope stowed inside the shroud. Reference 4 by Stahl et al. provides a 
description of this EDRM. It provides an aperture larger than $8 \mathrm{~m}$ that is of benefit to exoplanet science and general astrophysics. This EDRM incorporates a coronagraph for exoplanet observations. At a conceptual design level, the 12.7 m EDRM approaches the need for providing the necessary optical system stability by leveraging depth in the axial dimension of the primary mirror structure.
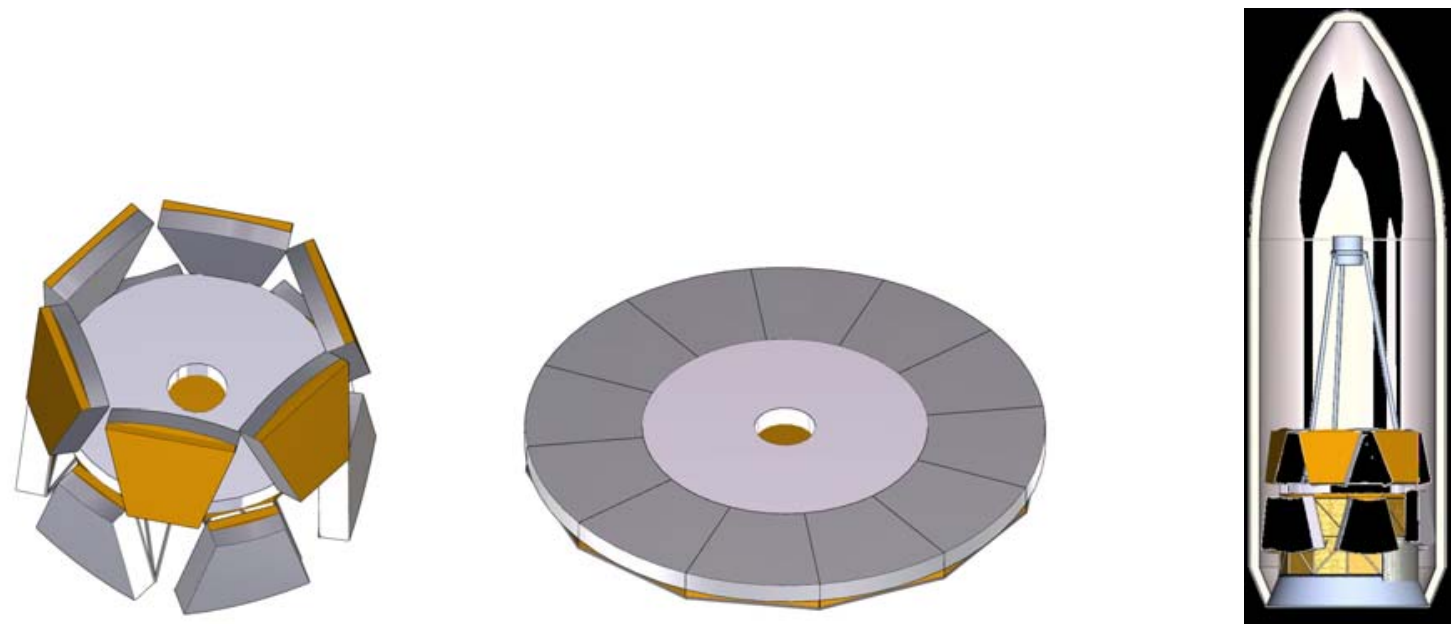

Figure 4. ATLAST design concept utilizing a $12.7 \mathrm{~m}$ segmented primary mirror.

The Scalable Segmented EDRM consists of a deployable primary mirror as illustrated in Figures 5 and 6. The aperture is scalable, meaning that its architecture supports adding more rings of segmented mirrors to increase the aperture in response to the refinement of the science requirements. The $9.2 \mathrm{~m}$ configuration has been validated to fit within a five meter launch vehicle fairing, which is an industry standard. This EDRM gives ATLAST immediate substantiation to the 2020 Decadal Survey that it is compatible with the flight proven Delta IV Heavy launch vehicle, as well as the Falcon Heavy and SLS which are currently in development. As discussed in the launch vehicle section, this is a benefit in controlling launch vehicle programmatic risks and associated costs. This EDRM will be described in more detail below.
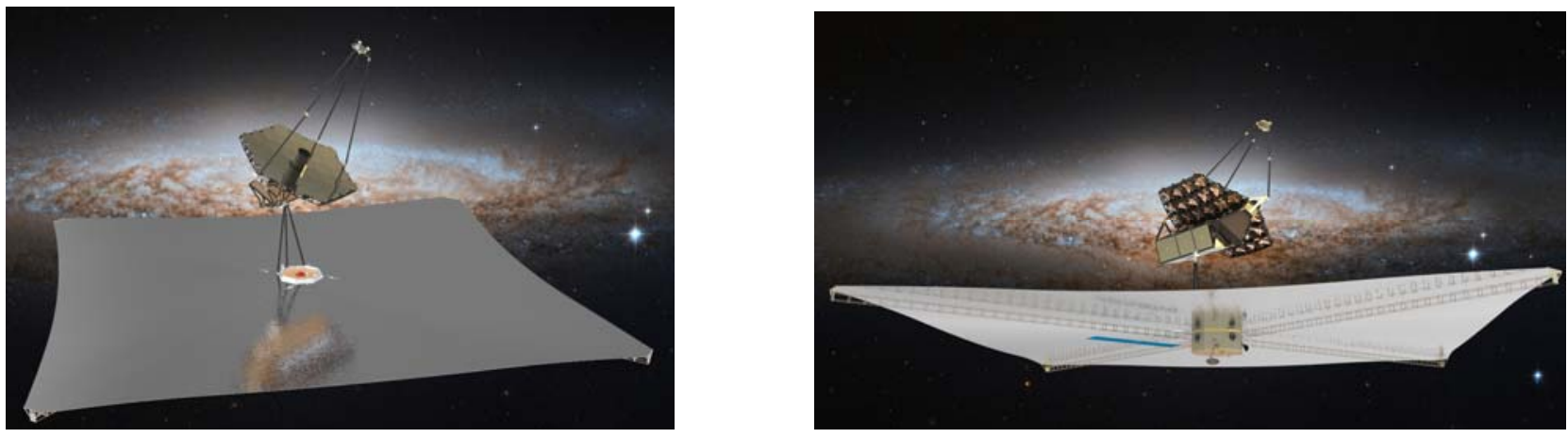

Figures 5 and 6. ATLAST Scalable segmented design concept utilizing a $9.2 \mathrm{~m}$ segmented primary mirror.

\section{SYSTEMS CONSIDERATIONS}

The science objectives associated with the exoplanet science portion of the mission are challenging. The mission system must provide simultaneous solutions for multiple science parameters affecting the end to end telescope-coronagraph system. These simultaneous solutions are being worked as part of the development of coronagraph design candidates. Some of the key science parameters involved include inner working angle (IWA), throughput, band pass, contrast, survey integration time, aperture and yield. Yield is a key performance parameter for the survey of potentially lifebearing exo-planets. Aperture is perhaps the most easily recognized parameter associated with the system, but it must be taken into account with the simultaneous solution space for the other parameters. The study of science system parameters that affect yield is ongoing and evolving over time. The scalability aspect of the Scalable Segmented EDRM is a benefit because it provides an opportunity to optimize aperture size in balance with multiple other key performance parameters, 
while not having to change the basic mission architecture. Additional rings of mirror segments can be added to the primary mirror while maintaining the same basic design approach. The Scalable Segmented EDRM provides ATLAST with immediate compatibility with an existing launch vehicle, and an opportunity to close on all the science parameters without trying to maximize aperture until it is clear where margin and opportunities in the overall system are for efficiently accomplishing that. This approach enables a cost effective and well balanced mission design process in this early stage where there are naturally many uncertainties and system requirements yet to be refined or quantified.

The coronagraph will place stringent wave front error stability requirements on the end to end optical system. This in turn will drive requirements for mechanical dynamics and jitter stability, as well as thermal stability. Studies are underway to analyze the extent to which jitter can be suppressed and managed, as well as the effect of thermal control and stability on wave front error of the primary mirror. As the candidate coronagraph designs mature, their individual stability requirements will become better understood. There is at least one coronagraph design (reference [5]) in work that may offer an easing of stability requirements of the telescope system. These coronagraph developments are being monitored as progress is being made. In the meantime efforts are underway on observatory conceptual designs that seek to maximize the amount of system stability that can practically be achieved.

\section{MECHANICAL DESIGN AND DYNAMIC STABILITY}

The ATLAST Scalable Segmented EDRM set out to validate that a large deployed telescope aperture can readily be stowed inside a 5 meter diameter launch vehicle fairing. 5 meter diameter fairings are an industry standard and are the most routinely available large fairing. The Delta IV Heavy, SLS, and Falcon Heavy launch vehicles all can support 5 meter fairings. After demonstrating an effective design concept that enables the science objectives with a 5 meter fairing, larger fairings provide an opportunity to enhance the mission and increase beneficial margin. Computer aided design (CAD) mechanical engineering studies have validated the feasibility of stowing and deploying a $9.2 \mathrm{~m}$ diameter primary mirror aperture in a 5 meter fairing. This version of the Scalable Segmented EDRM will be referred to here as the $9.2 \mathrm{~m}$ EDRM. A recent evolution of the concept appears in Figures 7 and 8. The deployment architecture leverages the primary mirror deployment work accomplished under JWST. Whereas JWST uses two deployable wings (one on each side of the primary mirror aperture), the 9.2m EDRM uses three deployable wings on each side of a central strip of mirror segments.
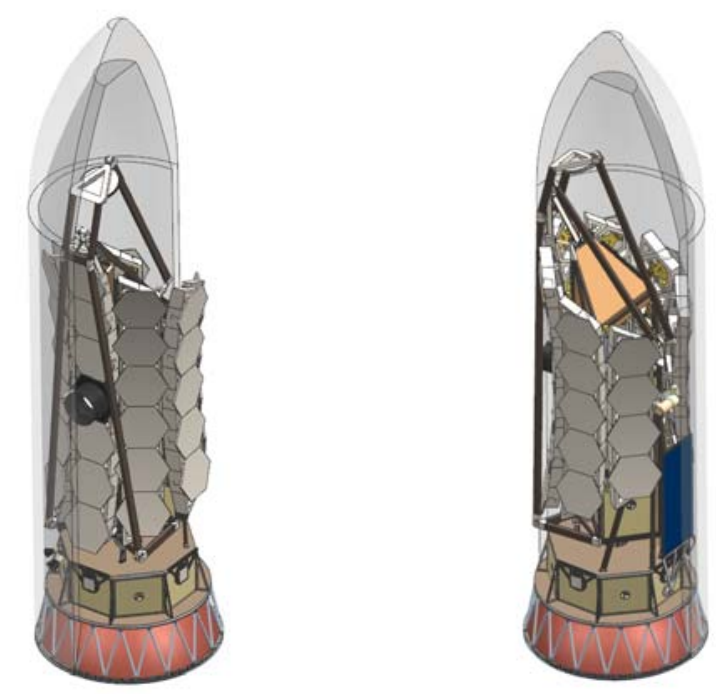

Figures 7 and 8 - ATLAST 9.2m Primary Mirror in a 5 m Fairing

Conceptual mechanical design work has been done on the observatory, with particular emphasis on the structure of the primary mirror backplane. Figure 9 shows the current evolution of the backplane design concept. Preliminary structural analysis indicates a first mode of $7.5 \mathrm{~Hz}$, which meets its design target. Figure 10 illustrates the shape of the first mode at an exaggerated scale to make it visible. 


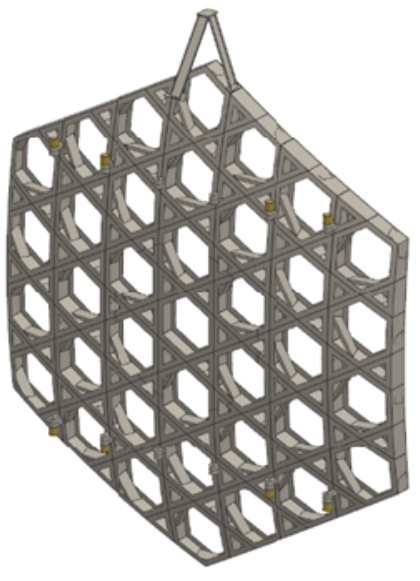

Figure 9 - Backplane Design - Spring 2015

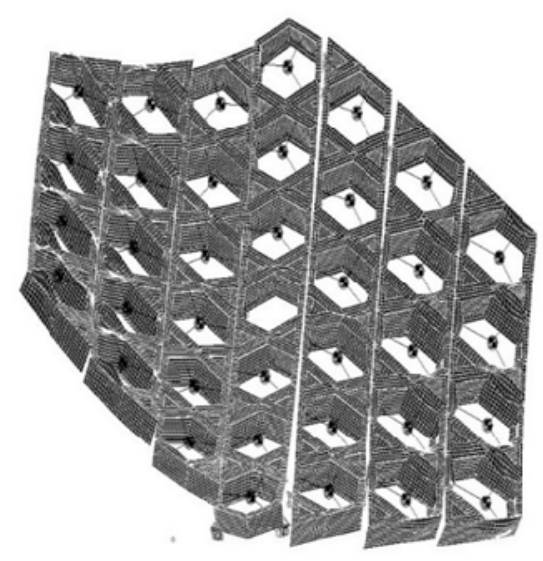

Figure 10 - 7.5 Hz First Primary Mirror Mode

Based on the results of preliminary analyses, the conceptual design for the 9.2m EDRM primary mirror backplane has been undergoing an iterative process of development and refinement. Originally, the ATLAST primary mirror backplane was represented as a simple shell. This allowed the team to quickly evaluate backplane configurations (most notably, backplane depth) with respect to the 5 meter diameter class fairings. Figure 11 below, shows the current concept iteration stowed inside the fairing. Primary mirror wing hinge locations, individual cell dimensions (See Figure 9 above) and a number of other gross dimensional parameters were evaluated for packaging options. As those trade studies matured, input from the analysis team enabled refinements to the mechanical design.

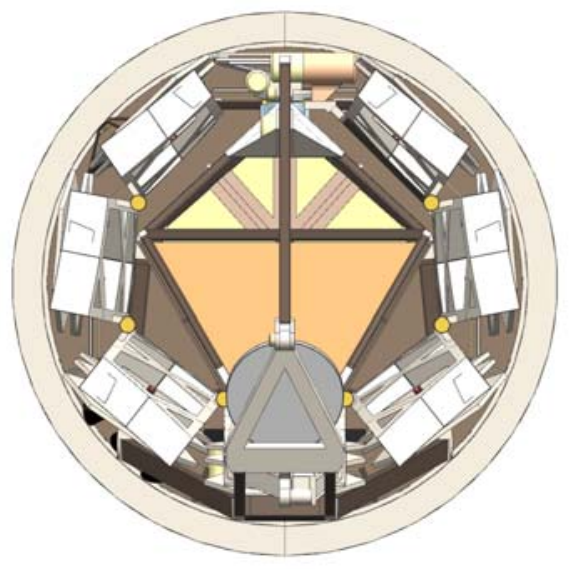

Figure 11 - Iterated concept of ATLAST stowed in 5 meter fairing

Early integrated modeling of the observatory has focused primarily on the stability of the primary and secondary mirrors and associated structures which are the main drivers for stability. The disturbance source was represented by a disturbance model of a commercial reaction wheel that includes static and dynamic imbalances. The performance of a representative and realizable non-contact dynamic disturbance isolation system was modeled and included in the analysis. Figure 12 below illustrates a preliminary analysis of wave front error (WFE) of the 9.2 m primary mirror as a function of reaction wheel speed. The result shown is for a worst case one wheel response. The initial indications of the preliminary modelling are consistent with the feasibility of a primary mirror being stiff enough to support operation with a coronagraph. Most of the primary mirror responses fall below the 10 picometer $(\mathrm{pm})$ level over a large range of wheel speeds. Continued conceptual design and development work will be applied to suppress and manage the effects of peak responses. In addition, guidance, navigation and control algorithms can be enhanced to avoid any residual peak resonances to help provide robust wheel speed ranges where 10pm stability performance can be achieved. 


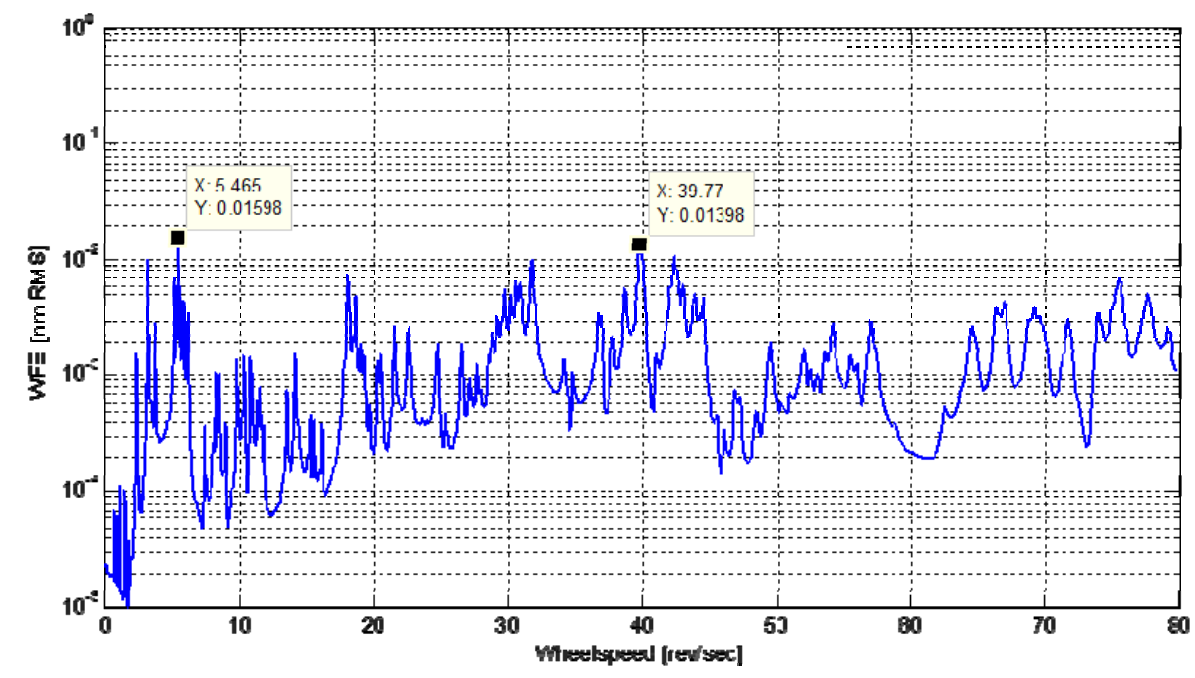

Figure 12 - Primary Mirror WFE vs. Reaction Wheel Speed

These are only preliminary results and more detailed modeling will be carried out with more mature models. Future improvements include adding the effects of the science payload boom and gimbal structure, along with a model uncertainty factor (MUF). Also, a key assumption thus far is that the structures will respond linearly at levels of disturbance relevant to picometer wave front error control. This will be examined more closely in future analysis supported by inputs from test data. It may turn out that non-linearity actually improves performance as energy is absorbed by the interfaces. Iteration on the stability analysis will continue and the conceptual design will continue to evolve in order to achieve optimal dynamic stability.

\section{THERMAL STABILITY}

Preliminary thermal stability analysis has focused on the feasibility of achieving wave front error consistent with picometer stability at the individual mirror segment level. Park and Eisenhower at SAO have carried out thermal analysis on a mirror segment that examines the ability to control wave front error with a high precision active heater control. Initial results are encouraging, and in the future the intent is to extend the analysis to include the backplane. This work is described in reference 6 .

\section{COST CONTROL}

ATLAST is an ambitious mission that sets out with two immediate priorities: First, enable revolutionary science that unites the science community, and second, control cost and cost uncertainty. Even this early in the conceptual formulation phase a strong foundation for cost control is being built into the mission. Many of the sections above provide examples of how cost control is being incorporated into the mission design at its inception. This section expands on the topic more.

A key part of the early work on ATLAST/LUVOIR has been identifying key technologies that enable the mission. These are documented in the ATLAST/LUVOIR Technology Development Plan. The ATLAST team is actively pursuing early funding for these key technologies. Early technology work reduces risk and can enable cost reductions over the project lifecycle.

The observatory with largest telescope aperture developed thus far for use in space is JWST. The ATLAST/LUVOIR Scalable Segmented EDRM is designed to be substantially less costly than simple extrapolation from, for example, the cost of JWST.

Unlike JWST, ATLAST/LUVOIR is non-cryogenic, thus making unnecessary the complex thermal designs, technologies, and integration and test associated with cryogenic performance. Cryogenic performance requirements drive costs through all phases of the development of the observatory - design, fabrication, and environmental test through all levels of assembly including observatory flight qualification. ATLAST will avoid this cost driving architecture. 
NASA's experience in the development of previous facility-class observatories demonstrates processes and practices that enable cost control, and provide examples of practices to avoid. Emphasis on the control of costs and cost uncertainty from the inception of ATLAST seeks to leverage these lessons learned along with using established best management practices. An example of this is placing heavy emphasis on using flight proven, TRL-9 technologies and components unless an element less mature is required to enable a significant leap in capability for meeting the mission science objectives. Another example is insisting on a room temperature telescope to avoid the significant cost and schedule impacts associated with flight qualifying a cryogenic telescope. Cost drivers like telescope temperature are being managed in concert with the science objectives of the mission. Unlike JWST, a cryogenic telescope is not fundamental to enabling ATLAST mission science. Studies are already being undertaken to characterize what science objectives in the near infra-red could be enabled with a passively cooled instrument working together with the room temperature telescope.

The ATLAST Scalable Segmented EDRM incorporates features which are beneficial for cost control. This conceptual design uses a segmented primary mirror that uses parallel processing paths, makes use of economies of scale and has high technology readiness. With regard to schedule for fabrication of the segmented telescope, the parallelization of the segmented system and leveraging existing facilities can enable advantages for shortening the critical path schedule.

The ATLAST segmented scalable EDRM builds upon designs, hardware, mechanisms, personnel, ground support equipment, facilities, and experience with JWST and other segmented optical systems. This enables ATLAST to immediately leverage these mature aspects of a segmented space telescope program from its inception. The scope of these advantages is significant. For example even in the conceptual formulation phase ATLAST is already taking advantage of the mature environmental requirements specifications for the Sun-Earth L2 orbit environment that have previously been developed under JWST. Having this degree of maturity in environmental specifications drives out uncertainty in this aspect of the requirements, accelerates the maturity of design concepts, and drives down risk early in the program.

ATLAST is designing for compatibility with multiple launch vehicles and fairing configurations. These include the Delta IV Heavy, SLS, and Falcon Heavy launch vehicles. ATLAST will have solutions compatible with 5 meter diameter fairings standard to all these launch vehicles, as well as the 8.4 and 10 meter diameter fairings that may become available with the SLS vehicle. This flexibility manages launch vehicle risk and the potential costs associated with any particular vehicle becoming unavailable in the mid to late 2020s.

\section{REFERENCES}

[1] J. Dalcanton, S. Seager, et al., From Cosmic Birth to Living Earths: The Future of UVOIR Space Astronomy, published by the Association of Universities for Research in Astronomy, www.hdstvision.org/report/ (2015).

[2] M. R. Bolcar, et al., "Technology Development for the Advanced Technology Large Aperture Space Telescope (ATLAST) as a Candidate Large UV-Optical-Infrared (LUVOIR) Surveyor", paper 9602-8, SPIE Optics + Photonic 2015.

[3] H. Philip Stahl, et al., "ATLAST-8 Mission concept study for 8-meter Monolithic UV/Optical Space Telescope”, Space Telescopes and Instrumentation 2010: Optical, Infrared, and Millimeter Wave, Proceedings of SPIE Volume 7731 (2010).

[4] H. Philip Stahl, et al., "ATLAST-12 Observatory Preliminary Design Study”, chart package, (2015).

[5] R. G. Lyon, et. al., "Phase-Occultation Nulling Coronagraph," submitted to the Astrophysics Journal, arXiv:1504.05747 (2015).

[6] "ATLAST ULE Mirror Segment Performance Analytical Predictions Based on Thermally Induced Distortions" (Eisenhower, Park, et al.). 